\title{
Avaliação econômica da atenção em saúde mental baseada no financiamento público: Uma revisão integrativa
}

\author{
Economic evaluation of publicly funded mental health care: An integrative review \\ Evaluación económica de la atención de salud mental financiada con fondos públicos: Una revisión \\ integradora
}

Recebido: 12/06/2021 | Revisado: 19/06/2021 | Aceito: 20/06/2021 | Publicado: 05/07/2021

Raiane Jordan da Silva Araújo

ORCID: https://orcid.org/0000-0001-9443-1909

Universidade Federal de Alagoas, Brasil

E-mail: raianejsa@hotmail.com

Mariana da Silva Pacheco

ORCID: https://orcid.org/0000-0002-7599-8157

Universidade Federal de Alagoas, Brasil

E-mail: maari_pacheco@hotmail.com

Willienay Tavares Costa

ORCID: https://orcid.org/0000-0003-4120-7959

Universidade de Pernambuco, Brasil

E-mail: naytavareswt@gmail.com

Claudio Torres de Miranda

ORCID: https://orcid.org/0000-0002-9602-6736 Universidade Federal de Alagoas, Brasil

E-mail: mirandaclaudio@gmail.com

Wagner Silva Ribeiro

ORCID: https://orcid.org/0000-0002-9602-6736 London School of Economics and Political Science, Reino Unido

E-mail:W.Silva-Ribeiro@1se.ac.uk

Sara Evans-Lacko

ORCID: https://orcid.org/0000-0003-4691-2630 London School of Economics and Political Science, Reino Unido

E-mail: s.evans-lacko@1se.ac.uk

Maria Cicera dos Santos de Albuquerque

ORCID: https://orcid.org/0000-0002-5230-3447

Universidade Federal de Alagoas, Brasil

E-mail: cicera.albuquerque@eenf.ufal.br

Verônica de Medeiros Alves

ORCID: https://orcid.org/0000-0002-4343-2941

Universidade Federal de Alagoas, Brasil

E-mail: veronica.alves@eenf.ufal.br

\section{Resumo}

Globalmente, as despesas públicas relacionadas ao cuidado em saúde mental têm sido alvo de diferentes estudos de avaliação econômica, permitindo a compreensão do cenário que engloba a efetividade e o custeio da assistência. Objetivo: Analisar o custo da assistência em saúde mental no contexto do financiamento público. Metodologia: Revisão integrativa, sem delimitação temporal. Utilizado a estratégia PICO para combinações de termos e utilização do operador booleano "AND”, abrangendo bases de dados: Medline via PubMed, LILACS, EMBASE, Web of Science, SCOPUS e COCHRANE LIBRARY. O nível de evidência e a qualidade metodológica de cada estudo foram avaliados. Utilizouse o método PRISMA. Resultados: Encontrados 266 estudos. Após avaliação por pares, 16 selecionados para compor a revisão. A maioria (75\%) das pesquisas foi realizada em países europeus, na perspectiva de provedores de serviços, principalmente, no contexto hospitalar e comunitário. Os estudos envolviam investigações relacionadas aos custos indiretos e diretos, médico hospitalares e não médico hospitalares: de serviços, de intervenções medicamentosas, de transtornos mentais com destaque para esquizofrenia. Foram mensurados através da estatística descritiva, e analisados pelo método de microcusteio (43,7\%), com variação temporal de 1 a 36 meses. Quanto aos achados principais destacase o impacto no uso dos serviços e no tratamento farmacológico, bem como a descrição de alguns valores médios per capita mensal que foram distintos na avaliação econômica realizada. Conclusão: Este estudo de revisão integrativa traz informações que podem implicar em condutas de gestão e clínicas que podem melhorar e otimizar aspectos do custeio público da assistência em saúde mental.

Palavras-chave: Custos diretos de serviços; Avaliação em saúde; Serviços de saúde mental; Custos e análise de custo; Efeitos psicossociais da doença. 


\begin{abstract}
Globally, public expenditures related to mental health care have been the target of different economic evaluation studies, allowing for an understanding of the scenario that encompasses the effectiveness and cost of care. Objective: To analyze the cost of mental health care in the context of public funding. Methodology: Integrative review, without temporal delimitation. Used the PICO strategy for combinations of terms and use of the Boolean operator "AND", covering databases: Medline via PubMed, LILACS, EMBASE, Web of Science, SCOPUS and COCHRANE LIBRARY. The level of evidence and methodological quality of each study were assessed. The PRISMA method was used. Results: 266 studies were found. After peer review, 16 selected to compose the review. Most (75\%) of the surveys were carried out in European countries, from the perspective of service providers, mainly in the hospital and community context. The studies involved investigations related to indirect and direct costs, hospital doctors and non-medical hospitals: services, drug interventions, mental disorders, with emphasis on schizophrenia. They were measured using descriptive statistics, and analyzed using the microcosting method (43.7\%), with a temporal variation from 1 to 36 months. As for the main findings, the impact on the use of services and pharmacological treatment stands out, as well as the description of some average monthly per capita values that were different in the economic evaluation carried out. Conclusion: This integrative review study provides information that can lead to management and clinical approaches that can improve and optimize aspects of public funding for mental health care.
\end{abstract}

Keywords: Service direct costs; Health evaluation; Mental health services; Cost and cost analysis; Psychosocial effects of illness.

\title{
Resumen
}

A nivel mundial, los gastos públicos relacionados con la atención en salud mental han sido objeto de diferentes estudios de evaluación económica, lo que permite comprender el escenario que engloba la efectividad y el costo de la atención. Objetivo: Analizar el costo de la atención en salud mental en el contexto de la financiación pública. Metodología: Revisión integrativa, sin delimitación temporal. Se utilizó la estrategia PICO para combinaciones de términos y uso del operador booleano "AND", cubriendo bases de datos: Medline vía PubMed, LILACS, EMBASE, Web of Science, SCOPUS y COCHRANE LIBRARY. Se evaluó el nivel de evidencia y la calidad metodológica de cada estudio. Se utilizó el método PRISMA. Resultados: Se encontraron 266 estudios. Después de la revisión por pares, 16 fueron seleccionados para redactar la revisión. La mayoría (75\%) de las encuestas se realizaron en países europeos, desde la perspectiva de los proveedores de servicios, principalmente en el contexto hospitalario y comunitario. Los estudios involucraron investigaciones relacionadas con costos indirectos y directos, médicos hospitalarios y hospitales no médicos: servicios, intervenciones farmacológicas, trastornos mentales, con énfasis en la esquizofrenia. Se midieron mediante estadística descriptiva y se analizaron mediante el método de microcostes (43,7\%), con una variación temporal de 1 a 36 meses. En cuanto a los principales hallazgos, se destaca el impacto en el uso de servicios y tratamiento farmacológico, así como la descripción de algunos valores promedio mensuales per cápita que fueron diferentes en la evaluación económica realizada. Conclusión: este estudio de revisión integradora proporciona información que puede conducir a enfoques clínicos y de gestión que pueden mejorar y optimizar aspectos de la financiación pública para la atención de la salud mental.

Palabras clave: Costos de servicios directos, Evaluación de la salud, Servicios de salud mental, Costos y análisis de costos, Efectos psicosociales de la enfermedad.

\section{Introdução}

Globalmente os países gastam em média, apenas $2 \%$ de seus orçamentos de saúde em saúde mental, o que faz com que essa seja uma das áreas mais negligenciadas da saúde pública (OMS, 2020). Atualmente, as avaliações administrativas e financeiras em serviços de saúde têm sido constantes e apontam a relação existente entre aspectos econômicos e as dimensões epidemiológicas no custeio da doença (Itria et al, 2012).

No Brasil, a perspectiva do custo econômico com assistência pública em saúde mental acompanhou os momentos históricos vivenciados com a própria luta pela efetivação da Reforma da Atenção em Saúde Mental ao longo dos anos. Houve uma inversão dos gastos e de recursos hospitalares para os serviços comunitários por volta de 2006. Em 2017, houve uma retomada aos investimentos hospitalares, com os reajustes no valor de internações hospitalares. Mesmo diante de uma relevante expansão dos serviços comunitários, eles ainda carecem de financiamento adequado (Onocko-Campos, 2019).

Na compreensão sobre custos em saúde sobressai que a identificação, mensuração e valoração dos mesmos são realizados mediante procedimentos estruturados que visam delimitar uma trajetória coerente com o que se pretende compreender. Para alcançar e expandir este conhecimento é essencial a apropriação desta temática, através da propagação da educação em análise econômica e do desenvolvimento da gestão de custo nos serviços de saúde (Silva, Silva \& Pereira, 2016). 
Diante do exposto, torna-se relevante a realização deste estudo, que tem como objetivo: analisar o custo da assistência em saúde mental no contexto do financiamento público.

\section{Metodologia}

Trata-se de uma revisão da literatura do tipo Integrativa que buscam apresentar uma visão atual, holística e relevante para o assunto em discussão. Sendo realizada com rigor metodológico em cinco etapas: Identificação do problema, Busca na literatura, Avaliação de dados, Análise de dados e Apresentação da revisão (Whittemore \& Knafl, 2005).

A identificação do problema de pesquisa e a busca bibliográfica seguiram a utilização da metodologia proposta por Santos, Pimenta e Nobre (2007) conhecida como estratégia PICO: Problema (Serviços Públicos de Saúde Mental), Intervenção (Análise do Custo), Comparação (Não Aplicado) e Desfecho (Gestão Pública) como recurso facilitador para o acesso a melhor informação científica disponível.

Ressalta-se que, dependendo do método de revisão, não se emprega todos os elementos da estratégia PICO. Nesta revisão integrativa, o terceiro elemento, ou seja, a comparação, não foi utilizada. O uso dessa estratégia para formular a questão de pesquisa na condução de métodos de revisão possibilita a identificação de palavras-chave, as quais auxiliam na localização de estudos primários relevantes nas bases de dado (Fineout-Overholt \& Stillwell, 2011). Assim, a questão de pesquisa delimitada foi: "Quais são as evidências disponíveis na literatura sobre análise de custo da assistência em saúde mental em serviços públicos?"

A busca e a seleção dos estudos foram realizadas de forma a possibilitar a análise por pares. Ou seja, foram realizadas por três pessoas. Isso garante maior segurança e legitimidade na condução da revisão integrativa. O processo de busca e seleção foi descrito em fluxograma (Figura 1), orientado na estratégia PRISMA (Preferred Reporting Items for Systematic reviews and Meta-Analyses) que estabelece direcionamento nos estudos de revisão (Moher et al., 2009).

Figura 1. Fluxograma de seleção dos estudos.

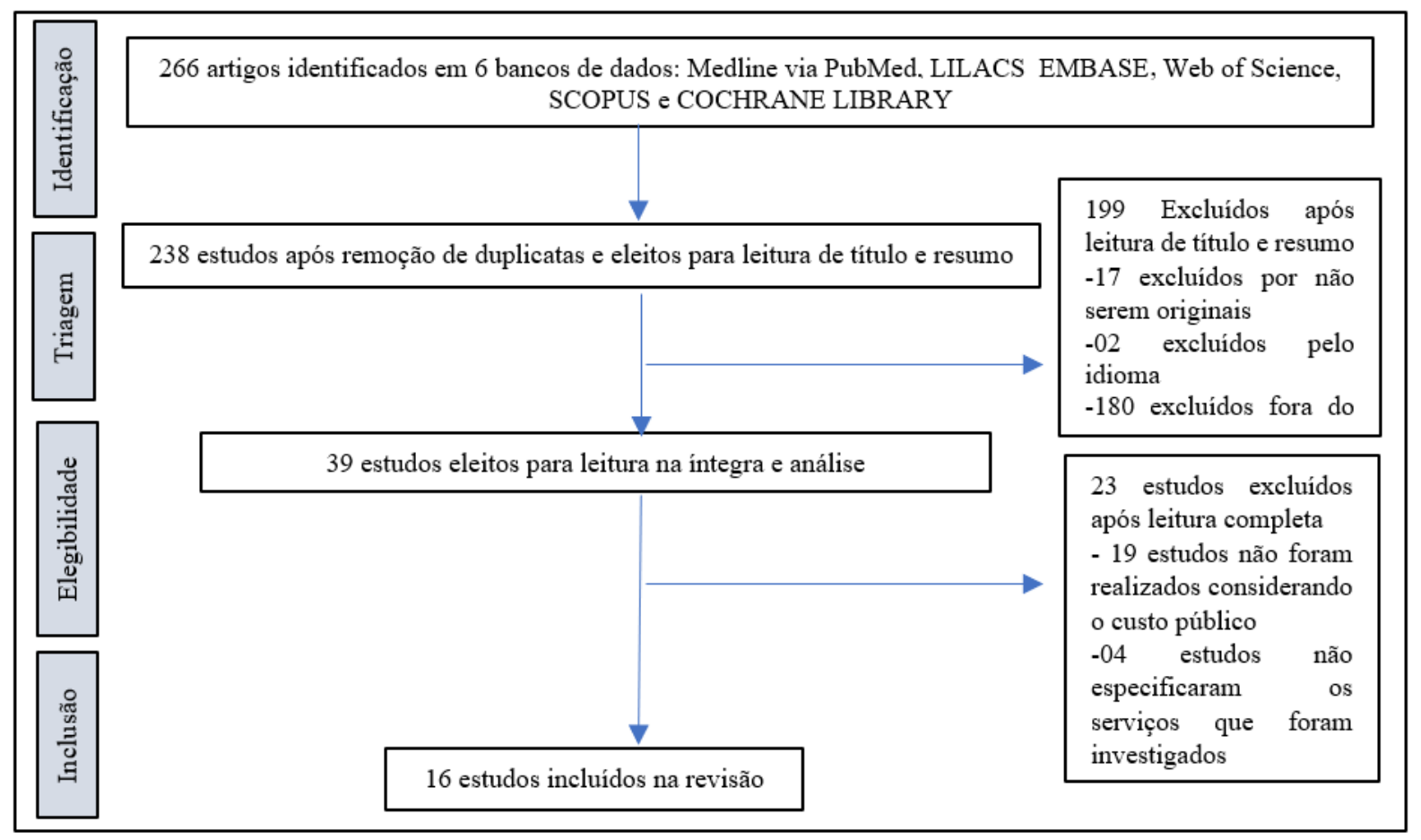

Fonte: Dados da própria pesquisa, baseado na metodologia PRISMA (Moher et al., 2009). 
Em maio de 2020, iniciou-se a combinação de termos de busca controlados (Mental Health Services, Direct Service Costs, Health Services Administration, Cost and Cost Analysis, Health Care Cost) com o operador booleano “AND” em seis bases de dados diferentes buscando encontrar o que estava disponível na literatura.

No segundo passo desta revisão foram apontados os Critérios de Inclusão para seleção dos artigos: a) publicações de estudos originais, b) relacionados ao custo da assistência em saúde mental em serviços públicos, c) e publicados nos idiomas: português, inglês ou espanhol. Não foi utilizado período de publicação como filtro de inclusão ou exclusão, sendo selecionado todo o período disponível na literatura.

Em relação aos Critérios de Exclusão foram eliminados: os artigos que não responderam à questão norteadora, estudos de revisão, editoriais e protocolos, pesquisas que mensuram o custo privado, de organizações não governamentais ou do paciente/família. Foram eliminados os estudos duplicados nas Bases de Dados.

O nível de evidência de cada estudo foi classificado conforme uma hierarquia composta por 7 níveis: 1 - o nível mais alto correspondente a estudos de revisão sistemática ou metanálise baseadas em ensaios clínicos randomizados controlados; 2 ensaios clínicos randomizados controlados; 3 - ensaios clínicos não-randomizados; 4 - coorte ou caso controle; 5 - revisão sistemática de estudos descritivos e qualitativos; 6 - estudo único descritivo ou qualitativo; e 7 - evidências provenientes de autoridades ou especialistas (Galvão, 2006).

A qualidade metodológica dos estudos incluídos na análise também foi levada em consideração e classificada conforme o modelo de Machotka, Kumar e Perraton (2009), através da avaliação de 12 itens relevantes que são somados e posteriormente o seu resultado é transformado em porcentagem exibindo a avaliação final (Tabela 1).

Tabela 1. Qualidade metodológica dos estudos.

\begin{tabular}{l|c|c|c|c|c|c|c|c|c|c|c|c|c|c}
\hline & \multicolumn{1}{|c}{ Critérios } \\
\hline Estudo & $\mathbf{1}$ & $\mathbf{2}$ & $\mathbf{3}$ & $\mathbf{4}$ & $\mathbf{5}$ & $\mathbf{6}$ & $\mathbf{7}$ & $\mathbf{8}$ & $\mathbf{9}$ & $\mathbf{1 0}$ & $\mathbf{1 1}$ & $\mathbf{1 2}$ & $\mathbf{S c o r e}$ & $\mathbf{\%}$ \\
\hline Wiersma et al (1991) & $\mathrm{Y}$ & $\mathrm{Y}$ & $\mathrm{Y}$ & $\mathrm{Y}$ & $\mathrm{Y}$ & $\mathrm{Y}$ & $\mathrm{NR}$ & $\mathrm{Y}$ & $\mathrm{Y}$ & $\mathrm{Y}$ & $\mathrm{Y}$ & $\mathrm{Y}$ & $11 / 12$ & 91,6 \\
\hline Becker \& Razzouk (2018) & $\mathrm{Y}$ & $\mathrm{Y}$ & $\mathrm{Y}$ & $\mathrm{Y}$ & $\mathrm{Y}$ & $\mathrm{Y}$ & $\mathrm{NA}$ & $\mathrm{NR}$ & $\mathrm{Y}$ & $\mathrm{Y}$ & $\mathrm{NA}$ & $\mathrm{Y}$ & $9 / 12$ & 75,0 \\
\hline Daltio, Mari \& Ferraz (2011) & $\mathrm{Y}$ & $\mathrm{Y}$ & $\mathrm{Y}$ & $\mathrm{Y}$ & $\mathrm{Y}$ & $\mathrm{Y}$ & $\mathrm{NA}$ & $\mathrm{NR}$ & $\mathrm{Y}$ & $\mathrm{Y}$ & $\mathrm{NA}$ & $\mathrm{Y}$ & $9 / 12$ & 75,0 \\
\hline Hui-Chih Chang et al (2012) & $\mathrm{Y}$ & $\mathrm{Y}$ & $\mathrm{Y}$ & $\mathrm{Y}$ & $\mathrm{Y}$ & $\mathrm{Y}$ & $\mathrm{NR}$ & $\mathrm{Y}$ & $\mathrm{Y}$ & $\mathrm{Y}$ & $\mathrm{Y}$ & $\mathrm{Y}$ & $11 / 12$ & 91,6 \\
\hline Ekman et al (2013) & $\mathrm{Y}$ & $\mathrm{Y}$ & $\mathrm{Y}$ & $\mathrm{Y}$ & $\mathrm{Y}$ & $\mathrm{Y}$ & $\mathrm{NA}$ & $\mathrm{Y}$ & $\mathrm{Y}$ & $\mathrm{Y}$ & $\mathrm{NA}$ & $\mathrm{Y}$ & $10 / 12$ & 83,3 \\
\hline Percudani et al (2004) & $\mathrm{Y}$ & $\mathrm{Y}$ & $\mathrm{Y}$ & $\mathrm{NR}$ & $\mathrm{Y}$ & $\mathrm{Y}$ & $\mathrm{NA}$ & $\mathrm{Y}$ & $\mathrm{Y}$ & $\mathrm{Y}$ & $\mathrm{NA}$ & $\mathrm{Y}$ & $9 / 12$ & 75,0 \\
\hline Medina et al (2018) & $\mathrm{Y}$ & $\mathrm{Y}$ & $\mathrm{Y}$ & $\mathrm{Y}$ & $\mathrm{Y}$ & $\mathrm{Y}$ & $\mathrm{NA}$ & $\mathrm{Y}$ & $\mathrm{Y}$ & $\mathrm{Y}$ & $\mathrm{NA}$ & $\mathrm{Y}$ & $10 / 12$ & 83,3 \\
\hline Tarricone et a (2000) & $\mathrm{Y}$ & $\mathrm{Y}$ & $\mathrm{Y}$ & $\mathrm{Y}$ & $\mathrm{Y}$ & $\mathrm{Y}$ & $\mathrm{NA}$ & $\mathrm{Y}$ & $\mathrm{Y}$ & $\mathrm{Y}$ & $\mathrm{NA}$ & $\mathrm{Y}$ & $10 / 12$ & 83,3 \\
\hline Lindner et al (2009) & $\mathrm{Y}$ & $\mathrm{Y}$ & $\mathrm{Y}$ & $\mathrm{Y}$ & $\mathrm{Y}$ & $\mathrm{Y}$ & $\mathrm{NR}$ & $\mathrm{NR}$ & $\mathrm{Y}$ & $\mathrm{Y}$ & $\mathrm{NR}$ & $\mathrm{Y}$ & $9 / 12$ & 75,0 \\
\hline Son et al(2019) & $\mathrm{Y}$ & $\mathrm{Y}$ & $\mathrm{Y}$ & $\mathrm{NR}$ & $\mathrm{Y}$ & $\mathrm{Y}$ & $\mathrm{NR}$ & $\mathrm{Y}$ & $\mathrm{Y}$ & $\mathrm{Y}$ & $\mathrm{NR}$ & $\mathrm{Y}$ & $9 / 12$ & 75,0 \\
\hline Tait et al. (2016) & $\mathrm{Y}$ & $\mathrm{Y}$ & $\mathrm{Y}$ & $\mathrm{Y}$ & $\mathrm{Y}$ & $\mathrm{Y}$ & $\mathrm{NR}$ & $\mathrm{Y}$ & $\mathrm{Y}$ & $\mathrm{Y}$ & $\mathrm{Y}$ & $\mathrm{Y}$ & $11 / 12$ & 91,6 \\
\hline Razzouk et al (2015) & $\mathrm{Y}$ & $\mathrm{Y}$ & $\mathrm{Y}$ & $\mathrm{Y}$ & $\mathrm{Y}$ & $\mathrm{Y}$ & $\mathrm{NA}$ & $\mathrm{Y}$ & $\mathrm{Y}$ & $\mathrm{Y}$ & $\mathrm{Y}$ & $\mathrm{Y}$ & $11 / 12$ & 91,6 \\
\hline Ruggeri et al.(2004) & $\mathrm{Y}$ & $\mathrm{Y}$ & $\mathrm{Y}$ & $\mathrm{Y}$ & $\mathrm{Y}$ & $\mathrm{Y}$ & $\mathrm{NR}$ & $\mathrm{Y}$ & $\mathrm{Y}$ & $\mathrm{Y}$ & $\mathrm{Y}$ & $\mathrm{Y}$ & $11 / 12$ & 91,6 \\
\hline Salize et al.(2009) & $\mathrm{Y}$ & $\mathrm{Y}$ & $\mathrm{Y}$ & $\mathrm{Y}$ & $\mathrm{Y}$ & $\mathrm{Y}$ & $\mathrm{Y}$ & $\mathrm{N}$ & $\mathrm{Y}$ & $\mathrm{Y}$ & $\mathrm{Y}$ & $\mathrm{Y}$ & $11 / 12$ & 91,6 \\
\hline Amaddeo et al.(1997) & $\mathrm{Y}$ & $\mathrm{Y}$ & $\mathrm{Y}$ & $\mathrm{Y}$ & $\mathrm{Y}$ & $\mathrm{Y}$ & $\mathrm{NA}$ & $\mathrm{Y}$ & $\mathrm{Y}$ & $\mathrm{Y}$ & $\mathrm{NA}$ & $\mathrm{Y}$ & $10 / 12$ & 83,3 \\
\hline Garattini et al. (2001) & $\mathrm{Y}$ & $\mathrm{Y}$ & $\mathrm{Y}$ & $\mathrm{Y}$ & $\mathrm{Y}$ & $\mathrm{Y}$ & $\mathrm{NA}$ & $\mathrm{Y}$ & $\mathrm{Y}$ & $\mathrm{Y}$ & $\mathrm{NA}$ & $\mathrm{Y}$ & $10 / 12$ & 83,3 \\
\hline
\end{tabular}

Nota: 1=Objetivo do estudo relatado; 2 =Literatura de base relevante; $3=$ Descrição da amostra; 4=Justificação do tamanho amostral; $5=$ Medidas de resultados confiáveis e válidas; $6=$ Descrição da intervenção; $7=$ Contaminação e co-intervenção; 8=Significância estatística; $9=$ Método (s) de análise apropriado (s); 10=Importância clínica; 11=desistências; 12=Conclusões apropriadas. N=não; NA=não aplicável; NR= não relatado; $\mathrm{Y}=\operatorname{sim}$

Fonte: Dados da própria pesquisa, baseado no modelo de Machotka, Kumar e Perraton (2009). 
Foram construídas planilhas com informações relacionadas a avaliação de cada revisor em cada fase de seleção, também foi construída uma ficha de extração para facilitar a aquisição dos dados de cada estudo. Em seguida, os dados foram compilados em tabelas com Matriz de Síntese com os principais achados.

\section{Resultados}

Incialmente foram identificados 266 artigos. Dentre eles, 28 foram excluídos por duplicação nas bases de dados. Mediante os critérios de inclusão e exclusão, a primeira triagem foi feita com a leitura de 238 títulos e resumos, restando 39 para avaliação crítica e leitura na íntegra. Ao final da revisão, 16 artigos foram direcionados para análise e discussão (Figura 1).

A apresentação dos resultados inerentes a produção deste estudo será exposta através de três categorias de dados: Dados gerais de identificação (Tabela 2), Dados Metodológicos (Tabela 3) e Principais Achados (Tabela 4), contemplados na Etapa de Análise de Dados e expostos em Matriz de Síntese.

Em relação aos dados gerais de identificação: houve variação entre 1991 a 2019, no período de publicação dos artigos, correspondendo a um recorte cronológico de 28 anos. Predominaram estudos realizados na Europa (58,3\%), embora também tenham sido contemplados estudos brasileiros (33,3\%) (Tabela 2).

Tabela 2. Matriz de Síntese dos dados gerais de identificação dos artigos selecionados no estudo.

\begin{tabular}{|c|c|c|c|}
\hline Citação & Titulo & Periódico & País \\
\hline Wiersma et al. (1991) & Costs and benefits of day treatment with community care for schizophrenic patients & $\begin{array}{c}\text { Schizophrenia } \\
\text { Bulletin }\end{array}$ & NLD \\
\hline $\begin{array}{l}\text { Becker \& Razzouk } \\
\text { (2018) }\end{array}$ & $\begin{array}{c}\text { Cost of a community mental health service: a retrospective study on a psychosocial } \\
\text { care center for alcohol and drug users in Sao Paulo }\end{array}$ & Sao Paulo Med J. & BRA \\
\hline $\begin{array}{c}\text { Daltio, Mari \& } \\
\text { Ferraz. } \\
\text { (2011) } \\
\end{array}$ & $\begin{array}{l}\text { Direct medical costs associated with schizophrenia relapses in health care services in } \\
\text { the city of Sao Paulo }\end{array}$ & Rev Saude Publica & BRA \\
\hline $\begin{array}{l}\text { Hui-Chih Chang et al. } \\
\text { (2012) }\end{array}$ & $\begin{array}{l}\text { A cost-consequence analysis of long-acting injectable risperidone in schizophrenia: } \\
\text { A one-year mirror-image study with national claim-based database in Taiwan }\end{array}$ & $\begin{array}{c}\text { Journal of } \\
\text { Psychiatric Research }\end{array}$ & TWN \\
\hline Ekman et al. (2013) & The societal cost of bipolar disorder in Sweden & $\begin{array}{c}\text { Soc Psychiatry } \\
\text { Psychiatr Epidemiol }\end{array}$ & SWE \\
\hline $\begin{array}{l}\text { Percudani et al. } \\
\text { (2004) }\end{array}$ & $\begin{array}{l}\text { Routine outcome monitoring in clinical practice: Service and non-service costs of } \\
\text { psychiatric patients attending a Community Mental Health Centre in Italy }\end{array}$ & European Psychiatry & ITA \\
\hline Medina et al. (2018) & $\begin{array}{c}\text { Costos médicos directos por intento de suicidio en pacientes del hospital mental de } \\
\text { Antioquia, Colombia }\end{array}$ & $\begin{array}{l}\text { Rev Panam Salud } \\
\text { Publica }\end{array}$ & $\mathrm{COL}$ \\
\hline $\begin{array}{l}\text { Tarricone et al. } \\
\text { (2000) }\end{array}$ & $\begin{array}{c}\text { Direct and indirect costs of schizophrenia in community psychiatric services in Italy: } \\
\text { The GISIES study }\end{array}$ & Health Policy & ITA \\
\hline Lindner et al. (2009) & $\begin{array}{c}\text { Avaliação econômica do tratamento da esquizofrenia com antipsicóticos no Sistema } \\
\text { Único de Saúde }\end{array}$ & Rev Saúde Pública & BRA \\
\hline Son et al. $(2019$ & $\begin{array}{l}\text { Understanding the direct and indirect costs of a first episode of psychosis program: } \\
\text { Insights from PAFIP of Cantabria, Spain, during the first year of intervention. }\end{array}$ & $\begin{array}{l}\text { Early Intervention in } \\
\text { Psychiatry }\end{array}$ & ESP \\
\hline Tait et al. (2016) & $\begin{array}{l}\text { Emergency department based intervention with adolescent substance users: } 10 \text { year } \\
\text { economic and health outcomes }\end{array}$ & $\begin{array}{c}\text { Drug and Alcohol } \\
\text { Dependence }\end{array}$ & AUS \\
\hline Razzouk et al (2015) & $\begin{array}{l}\begin{array}{l}\text { The impact of antipsychotic polytherapy costs in the public health care in Sao Paulo, } \\
\text { Brazil. }\end{array} \\
\end{array}$ & Plos one & BRA \\
\hline Ruggeri et al. (2004) & $\begin{array}{l}\text { A longitudinal evaluation of two-year outcome in a community-based mental health } \\
\text { service using graphical chain models. }\end{array}$ & $\begin{array}{l}\text { International Journal } \\
\text { of Methods in } \\
\text { Psychiatric Research }\end{array}$ & ITA \\
\hline Salize et al. (2009) & Cost of treatment of schizophrenia in six European countries. & $\begin{array}{l}\text { Schizophrenia } \\
\text { Research }\end{array}$ & $\begin{array}{c}\text { ESP, CHE, } \\
\text { SWE, } \\
\text { NLD, GBR, } \\
\text { DEU } \\
\end{array}$ \\
\hline Amaddeo et al. (1997) & The use of a case register to evaluate the costs of psychiatric care & $\begin{array}{l}\text { Acta Psychiatr } \\
\text { Scund }\end{array}$ & ITA \\
\hline Garattini et al. (2001) & Direct costs of schizophrenia in Italian community psychiatric services & Pharmacoeconomics & ITA \\
\hline
\end{tabular}

Fonte: Dados da própria pesquisa (2021).

Quanto aos dados metodológicos, foi possível identificar 4 perspectivas: a) predominância de provedores de serviço (81,3\%), principalmente no âmbito hospitalar (Daltio, Mari \& Ferraz, 2011; Chang et al, 2012; Medina et al, 2018; Tait et al, 2016; Razzouk et al, 2015) e comunitário (Becker \& Razzouk, 2018; Daltio, Mari \& Ferraz, 2011, Percudani et al, 2004; 
Tarricone et al, 2000; Razzouk et al, 2015; Ruggeri et al, 2004; Salize et al, 2009; Amaddeo et al, 1997) b) perspectiva do custo social (Ekman et al., 2013), c) no contexto do sistema de saúde brasileiro (Lindner et al, 2009) d) e italiano (Garattini et al, 2001) (Tabela 3).

Tabela 3. Matriz de Síntese dos dados metodológicos dos artigos selecionados no estudo.

\begin{tabular}{|c|c|c|c|c|c|c|}
\hline Citação & Perspectiva & $\begin{array}{l}\text { Sujeitos } e \\
\text { patologias }\end{array}$ & $\begin{array}{l}\text { Horizonte } \\
\text { temporal }\end{array}$ & $\begin{array}{c}\text { Custos } \\
\text { avaliados }\end{array}$ & Mensuração e método & Nivel \\
\hline $\begin{array}{l}\text { Wiersma et al. } \\
\quad \text { (1991) }\end{array}$ & $\begin{array}{l}\text { Provedor serviço: } \\
\text { GMH e RIAGG }\end{array}$ & $\begin{array}{l}\text { Adultos, } \\
\text { Esquizofrenia ou } \\
\text { psicose: } 34^{*}, 16^{* *}\end{array}$ & 24 meses & $\begin{array}{l}\text { Direto } \\
\text { MH } \\
\text { Direto } \\
\text { não MH } \\
\end{array}$ & Frequência, p, ANOVA, Microcusteio & 2 \\
\hline $\begin{array}{l}\text { Becker \& Razzouk } \\
\text { (2018) }\end{array}$ & $\begin{array}{l}\text { Provedor serviço: } \\
\text { CAPS-ad }\end{array}$ & $\begin{array}{l}\text { Adultos, } \\
\text { dependência } \\
\text { química: } 810\end{array}$ & 6 meses & $\begin{array}{l}\text { Direto } \\
\text { MH }\end{array}$ & $\begin{array}{l}\text { Frequência, média, mínimo e máximo, } \\
\text { variação Microcusteio }\end{array}$ & 6 \\
\hline $\begin{array}{l}\text { Daltio, Mari \& } \\
\text { Ferraz. }(2011)\end{array}$ & $\begin{array}{l}\text { Provedor serviço: } \\
\text { CAPS, Hospitais } \\
\text { público e } \\
\text { conveniado, }\end{array}$ & $\begin{array}{l}\text { Adultos, } \\
\text { Esquizofrenia: } 90 \\
\text { (prontuários) }\end{array}$ & 12 meses & $\begin{array}{l}\text { Direto } \\
\mathrm{MH}\end{array}$ & $\begin{array}{l}\text { Frequência, média, mínimo e máximo, desvio } \\
\text { padrão DMC = MCM + MCT + MCDR, } \\
\text { Microcusteio }\end{array}$ & 6 \\
\hline $\begin{array}{l}\text { Hui-Chih Chang } \\
\text { et al. }(2012)\end{array}$ & $\begin{array}{l}\text { Provedor serviço: } \\
\text { Ambulatório, } \\
\text { Emergência e } \\
\text { Hospital }\end{array}$ & $\begin{array}{l}\text { Adultos, } \\
\text { Esquizofrenia: } 184\end{array}$ & 12 meses & $\begin{array}{l}\text { Direto } \\
\text { MH }\end{array}$ & $\begin{array}{l}\text { Frequência, p, Wilcoxon sign-rank, média, } \\
\text { Técnica Mista }\end{array}$ & 4 \\
\hline $\begin{array}{l}\text { Ekman et al. } \\
\text { (2013) }\end{array}$ & Custo Social & $\begin{array}{l}\text { Adultos, Transtorno } \\
\text { Bipolar: } 611\end{array}$ & 36 meses & $\begin{array}{l}\text { Direto } \\
\mathrm{MH} \\
\text { Indireto }\end{array}$ & $\begin{array}{l}\text { Frequência, média, desvio padrão, } \\
\text { ANOVA,Shapiro-Wilks, Kruskal-Wallis, } \\
\text { Método do Capital humano }\end{array}$ & 6 \\
\hline $\begin{array}{l}\text { Percudani et al. } \\
\text { (2004) }\end{array}$ & $\begin{array}{l}\text { Provedor serviço: } \\
\text { CMHC }\end{array}$ & $\begin{array}{l}\text { Esquizofrenia } 50 \text { e } \\
\text { outras: } 70\end{array}$ & 3 meses & $\begin{array}{l}\text { Direto } \\
\mathrm{MH} \\
\text { Indireto }\end{array}$ & $\begin{array}{l}\text { Frequênciia, p, média, Mann-Whitney, } \\
\text { Kruskal-Wallis, Microcusteio }\end{array}$ & 6 \\
\hline $\begin{array}{l}\text { Medina et al. } \\
\quad(2018)\end{array}$ & $\begin{array}{l}\text { Provedor serviço: } \\
\text { Hospital Mental de } \\
\text { Antioquia }\end{array}$ & $\begin{array}{l}\text { Tentativa de } \\
\text { suicídio: } 248\end{array}$ & 36 meses & $\begin{array}{l}\text { Direto } \\
\text { MH }\end{array}$ & $\begin{array}{l}\text { Frequência, média, Kolgomorov-Smirnov, } \\
\text { Kruskal-Wallis, Microcusteio }\end{array}$ & 6 \\
\hline $\begin{array}{l}\text { Tarricone et al. } \\
\qquad(2000)\end{array}$ & $\begin{array}{l}\text { Provedor serviço: } \\
\text { CMHC (10) }\end{array}$ & $\begin{array}{l}\text { Adultos, } \\
\text { Esquizofrenia: } 100\end{array}$ & 12 meses & $\begin{array}{l}\text { Direto } \\
\mathrm{MH} \\
\text { Indireto }\end{array}$ & $\begin{array}{l}\text { Frequência, média, mediana, mínimo e } \\
\text { máximo, p, Microcusteio, Método do Capital } \\
\text { humano, }\end{array}$ & 6 \\
\hline $\begin{array}{l}\text { Lindner et al. } \\
\quad \text { (2009) }\end{array}$ & $\begin{array}{l}\text { Sistema Único de } \\
\text { Saúde }\end{array}$ & $\begin{array}{l}\text { Esquizofrenia: } 59 \\
\text { (prontuários) }\end{array}$ & 60 meses & $\begin{array}{l}\text { Direto } \\
\mathrm{MH}\end{array}$ & $\begin{array}{l}\text { Frequência, média, Técnica Mista, Modelo } \\
\text { Markov. }\end{array}$ & 6 \\
\hline Son et al. (2019) & $\begin{array}{l}\text { Provedor serviço: } \\
\text { Programa Hospitalar }\end{array}$ & $\begin{array}{l}\text { Psicose: } 157 \\
\text { (prontuários) }\end{array}$ & 12 meses & $\begin{array}{l}\text { Direto } \\
\mathrm{MH} \\
\text { Direto } \\
\text { não MH } \\
\text { Indireto } \\
\end{array}$ & $\begin{array}{l}\text { Frequência, média, desvio padrão, mínimo e } \\
\text { máximo, p, Shapiro-Wilk, Fisher, T-student, } \\
\text { Pearson, Técnica Mista. }\end{array}$ & 6 \\
\hline Tait et al. (2016) & $\begin{array}{l}\text { Provedor: Hospitais } \\
\text { Públicos (4) }\end{array}$ & $\begin{array}{l}\text { Adolescentes: } 67 * * \text {, } \\
60 * \text { dependência } \\
\text { química. }\end{array}$ & 120 meses & $\begin{array}{l}\text { Direto } \\
\mathrm{MH}\end{array}$ & Frequência, média, p, mediana, Macrocusteio & 2 \\
\hline $\begin{array}{l}\text { Razzouk et al } \\
\quad \text { (2015) }\end{array}$ & $\begin{array}{l}\text { Provedor serviço: } \\
\text { Hospitais, } \\
\text { ambulatório e } \\
\text { serviço comunitário }\end{array}$ & Adultos, 147 & 1 mês & $\begin{array}{l}\text { Direto } \\
\text { MH } \\
\text { Direto } \\
\text { não MH }\end{array}$ & $\begin{array}{l}\text { Frequência, média, mínimo e máximo, p, } \\
\text { Fischer, Microcusteio }\end{array}$ & 6 \\
\hline $\begin{array}{l}\text { Ruggeri et } \\
\text { al.(2004) }\end{array}$ & $\begin{array}{l}\text { Provedor serviço: } \\
\text { CMHS }\end{array}$ & Adultos, 178 & 3 meses & $\begin{array}{l}\text { Direto } \\
\text { MH }\end{array}$ & $\begin{array}{l}\text { Frequência, média, desvio padrão, Mann- } \\
\text { Whitney, p, Modelagem de Gauss }\end{array}$ & 4 \\
\hline Salize et al.(2009) & $\begin{array}{l}\text { Provedor serviço: } \\
\text { Serviço Comunitário }\end{array}$ & $\begin{array}{l}\text { Adultos, } \\
\text { Esquizofrenia: } 422\end{array}$ & 12 meses & $\begin{array}{l}\text { Direto } \\
\mathrm{MH}\end{array}$ & $\begin{array}{l}\text { Frequência, p, modelo de regressão linear, } \\
\text { Microcusteio }\end{array}$ & 2 \\
\hline $\begin{array}{l}\text { Amaddeo et } \\
\text { al.(1997) }\end{array}$ & $\begin{array}{l}\text { Provedor serviço: } \\
\text { CPS }\end{array}$ & Adultos, 706 & 12 meses & $\begin{array}{l}\text { Direto } \\
\mathrm{MH}\end{array}$ & $\begin{array}{l}\text { Frequência, média, desvio padrão, } p \text {, } \\
\text { microcusteio }\end{array}$ & 6 \\
\hline $\begin{array}{l}\text { Garattini et al. } \\
\quad \text { (2001) }\end{array}$ & $\begin{array}{l}\text { Serviço Nacional de } \\
\text { Saúde Italiano } \\
\text { (INHS) }\end{array}$ & $\begin{array}{l}\text { Adultos, } \\
\text { Esquizofrenia e } \\
\text { outros: } 677 \\
\end{array}$ & 2 meses & $\begin{array}{l}\text { Direto } \\
\text { MH }\end{array}$ & $\begin{array}{l}\text { Kruskal-Wallis, ANOVA, Fischer, R2, p, } \\
\text { Técnica Mista }\end{array}$ & 6 \\
\hline
\end{tabular}

*Grupo experimental, ** Grupo Controle, MH: médico-hospitalares, GMH: Hospital Geral Psiquiátrico, RIAGG: Regional Institute for Ambulatory Mental Health Care, CAPS: Centro de Atenção Psicossocial, CAPS-ad: Centro de Atenção Psicossocial Álcool e Outras Drogas, CMHC: Community Mental Health Centres, DMC: Custo Médio Direto, MCM: Custo Médio da Medicação, MCT: Custo Médio de Testes, MCDR: Custo Médio de Taxas Diárias. Nível: nível de evidência.

Fonte: Dados da própria pesquisa (2021).

No geral os estudos foram conduzidos com sujeitos adultos (91,6\%), e voltados para a investigação de intervenções específicas geralmente medicamentosas (Becker \& Razzouk, 2018; Chang et al, 2012; Razzouk et al, 2015), ou relacionados a 
um contexto patológico do custo da doença (Ekman et al., 2013; Medina et al, 2018; Son et al, 2018), direcionado principalmente à esquizofrenia (Wiersma et al, 1991; Daltio, Mari \& Ferraz, 2011; Tarricone et al, 2000; Salize et al, 2009; Garattini et al, 2001).

O recorte de tempo identificado nos estudos variou de 1 a 36 meses, sendo utilizada a média para representatividade mensal ou anual na descrição dos resultados. Foi descrito ainda, o custo direto médico hospitalar em todos os estudos. Houve utilização habitual da estatística descritiva para mensuração dos custos, e predominou o método de Microcusteio / bottom-up $(43,7 \%)$ (Tabela 3$)$.

Quanto aos achados principais destaca-se o impacto no uso dos serviços e no tratamento farmacológico, bem como a descrição de alguns valores médios per capita mensal que foram distintos em cada avaliação econômica realizada, devido a diferenças regionais, setoriais, período de realização da análise e público estudado (Tabela 4).

Tabela 4. Matriz de síntese dos principais achados apresentados nos artigos selecionados desta revisão integrativa.

\begin{tabular}{|c|c|}
\hline Citação & Resultados \\
\hline $\begin{array}{l}\text { Wiersma et al. } \\
\text { (1991) }\end{array}$ & O tratamento comunitário diário requer três vezes mais cuidado, porém, ainda é menos caro do que cuidados hospitalares $(1: 7,5)$. \\
\hline $\begin{array}{c}\text { Becker \& } \\
\text { Razzouk (2018) }\end{array}$ & $\begin{array}{l}\text { O custo total médio mensal do CAPS-ad foi de } \mathrm{R} \$ 64.017,54 \text {. Sendo que 56,5\% desse valor foi a despesa com a folha salarial dos } \\
\text { profissionais. Os custos médios per capita e por mês para cuidados intensivos e não intensivos foram: } \mathrm{R} \$ 668,34 \text { e } \mathrm{R} \$ 37,12 \text {. }\end{array}$ \\
\hline $\begin{array}{l}\text { Daltio, Mari e } \\
\text { Ferraz. (2011) }\end{array}$ & $\begin{array}{l}\text { O custo da recaída em Esquizofrenia por paciente foi de } \mathrm{R} \$ 8.167,58 \text { no hospital público estadual; R } \$ 4.605,46 \text { no centro comunitário de } \\
\text { saúde mental; e R } \$ 2.397,74 \text { no hospital conveniado ao SUS. A despesa com diária representou o maior impacto no custo total. }\end{array}$ \\
\hline $\begin{array}{c}\text { Hui-Chih } \\
\text { Chang et al. } \\
(2012)\end{array}$ & $\begin{array}{l}\text { O uso da risperidona injetável de ação prolongada em pacientes com Esquizofrenia produz impacto na utilização e custo dos serviços de } \\
\text { saúde mental. Reduzindo a utilização e custo com internações e aumentando a demanda e o custo em serviços comunitários. }\end{array}$ \\
\hline $\begin{array}{c}\text { Ekman et al. } \\
(2013)\end{array}$ & $\begin{array}{l}\text { O custo médio anual para o tratamento do Transtorno Bipolar por paciente foi de } € 28.011 \text {. Custos indiretos devido a licença médica e } \\
\text { aposentadoria precoce representou } 75 \% \text {. Custos de internação } 13 \% \text {, ambulatório custa } 8 \% \text {, produtos farmacêuticos } 2 \% \ldots\end{array}$ \\
\hline $\begin{array}{l}\text { Percudani et al. } \\
(2004)\end{array}$ & $\begin{array}{l}\text { Os custos médios per capita e por mês para cuidados de pacientes com Ezquizofrenia é €795.7 Emquamto que para outros transtornos } \\
\text { mentais foi de } € 379.4 \text {. No contexto geral o custo com cuidados comunitários foi maior do que com internações hospitalares. }\end{array}$ \\
\hline $\begin{array}{l}\text { Medina et al. } \\
\text { (2018) }\end{array}$ & $\begin{array}{l}\text { Os custos médios médicos diretos com cuidados de saúde aumentam significativamente em relação ao número de tentativas de suicídio, } \\
\text { com custo médio hospitalar per capita USD3.267. }\end{array}$ \\
\hline $\begin{array}{l}\text { Tarricone et al. } \\
(2000)\end{array}$ & $\begin{array}{l}\text { Mais da metade dos custos diretos totais foi atribuído a Intervenções do CMHC (ITL760.231). Os custos médios anuais de esquizofrenia } \\
\text { por paciente ascenderam a quase ITL } 50 \text { milhões: } 30 \% \text { para custos diretos e } 70 \% \text { para custos indiretos. }\end{array}$ \\
\hline $\begin{array}{l}\text { Lindner et al. } \\
\text { (2009) }\end{array}$ & $\begin{array}{l}\text { No modelo de Markov, a alternativa mais custo-efetiva foi a utilização de risperidona e haloperidol antes de olanzapina. O custo para } \\
\text { manter um paciente internado em um hospital psiquiátrico de Florianópolis por um período de } 22 \text { dias foi de US } \$ 317,07 \text { e o custo por } \\
\text { visita ao psiquiatra foi de US } \$ 3,67 \text {. }\end{array}$ \\
\hline $\begin{array}{l}\text { Son et al. } \\
(2019)\end{array}$ & $\begin{array}{l}\text { Em média, os custos totais durante o primeiro ano no tratamento da Psicose foram de } € 48353,51 \text { por paciente, com custos diretos de } \\
\text { saúde sendo } € 13729,47(28,39 \%) \text {, custos diretos não médicos sendo } € 08,6(0,22 \%) \text {, e custos indiretos } € 34515,44(71,39 \%) \text {. }\end{array}$ \\
\hline $\begin{array}{l}\text { Tait et al. } \\
\text { (2016) }\end{array}$ & $\begin{array}{l}\text { A intervenção não reduziu significativamente os custos médios gerais de saúde por paciente (intervenção } \$ 58746 \text { versus controle } \$ 64833 \text {, } \\
\mathrm{p}=0,800 \text { ). Da mesma forma, não houve diferença significativa nos custos associados a hospitalizações, serviços ambulatoriais de saúde } \\
\text { mental, ou farmacoterapias com opiáceos. }\end{array}$ \\
\hline $\begin{array}{c}\text { Razzouk et al } \\
(2015)\end{array}$ & $\begin{array}{l}\text { Os custos diretos totais mensais dos cuidados de saúde pública (cuidados de saúde, medicamentos e alojamento) foi: } \mathrm{R} \$ 529.425,26 \text {. } \\
\text { Deste, } \mathrm{R} \$ 35.153,03 \text { foram custos com medicamentos, sendo } \mathrm{R} \$ 31.658,29 \mathrm{com} \quad \text { antipsicóticos. }\end{array}$ \\
\hline $\begin{array}{l}\text { Ruggeri et al } \\
(2004)\end{array}$ & $\begin{array}{l}\text { Os custos médios diários foram de } € 13,85 \text {. Custo médio total por paciente no período de acompanhamento foi } € 10778,53 \text {. Destes, } 40,1 \% \\
\text { foram custos de internamento, e os demais custos ambulatoriais. }\end{array}$ \\
\hline $\begin{array}{l}\text { Salize et al. } \\
(2009)\end{array}$ & $\begin{array}{l}\text { Os custos dos cuidados de saúde mental foram mais baixos em Granada (Espanha) e mais altos em Zurique (Suíça), onde os custos totais } \\
\text { foram mais de dez vezes maior. }\end{array}$ \\
\hline $\begin{array}{l}\text { Amaddeo et al. } \\
(1997)\end{array}$ & $\begin{array}{l}\text { Os custos são significativamente maiores para pacientes com diagnóstico de esquizofrenia (17,2 milhões de liras ao ano) e distúrbios } \\
\text { relacionados do que para pacientes pertencentes ao outro grupo de diagnóstico }\end{array}$ \\
\hline $\begin{array}{l}\text { Garattini et al. } \\
\text { (2001) }\end{array}$ & O custo direto médio de pacientes com esquizofrenia no período de 2 meses de observação foi de 2234475 liras italianas. \\
\hline
\end{tabular}

Fonte: Dados da própria pesquisa (2021).

\section{Discussão}

Considerando como ponto de partida o estudo de Wiersma et al (1991) como o mais antigo incluído nesta revisão e associando ao movimento internacional pela reforma psiquiátrica, observa-se uma inclinação das pesquisas científicas identificadas no estudo de Daltio, Mari e Ferraz (2011) e Ruggeri et al (2004) para legitimar economicamente a viabilidade dos serviços comunitários de saúde mental em detrimento das internações hospitalares.

Neste aspecto, dois provedores de serviços merecem destaque na produção de dados relativos aos custos: CAPS e 
CMHS, por fornecerem o cuidado em saúde mental de forma pública e voltado para a comunidade. Embora estejam inseridos em sistemas de saúde nacional e internacional respectivamente, ambos foram analisados por mensuração, métodos semelhantes e clareza na delimitação dos custos envolvidos (Wiersma et al, 1991; Daltio, Mari \& Ferraz, 2011, Becker \& Razzouk, 2018; Percudani et al, 2004; Tarricone et al, 2000; Riggeri et al, 2004; Salize et al, 2009).

Os serviços hospitalares que oferecem internação psiquiátrica produzem maior valor de custo direto médico hospitalar, devido às diárias que demandam impacto considerável no custo total (Wiersma et al, 1991; Daltio, Mari \& Ferraz, 2011). Estudo mostra uma exceção compreensível relacionada ao uso da Risperidona injetável, em relação ao custo-benefício, pois, quando o paciente se encontra estável sem necessidade de internação, ele possivelmente utilizará mais o serviço comunitário do que o hospital (Chang et al, 2012).

Os valores do custo direto e indireto não foram comparados nesta análise uma vez que foram realizados em diferentes períodos e regiões, apresentados em diferentes moedas (Euro, Real, Dólar, Libras). E mesmo quando realizados com populações e países economicamente semelhantes, ainda assim houve variação de custos (Salize et al, 2009). Porém, é possível generalizar que o custo indireto foi maior que o custo direto quando avaliados em um mesmo estudo (Ekman et al, 2013; Tarricone et al, 2000).

Isto pode ser explicado pelo fato de que o custo indireto representa impacto social com maior proporção devido a perda da produtividade laboral, enquanto o custo direto está associado às despesas provenientes da assistência em saúde e está classificado em: médico hospitalar e não médico hospitalar (Silva, Silva \& Pereira, 2016).

O tamanho amostral relatado nos estudos teve variação entre 50 a 810 usuários dos respectivos serviços ou intervenções e foi imprescindível para o alcance dos valores financeiros apontados através de estratégias de identificação do custo médio (Daltio, Mari \& Ferraz, 2011). Através do consumo por indivíduo, é possível inferir medidas de tendência central para um determinado período (Silva, Silva \& Pereira, 2016).

Para avaliação das intervenções, os sujeitos da pesquisa foram selecionados por randomização (Wiersma et al, 1991; Tait et al, 2016; Salize et al, 2009) ou pela totalidade de usuários atendidos (Medina et al, 2018). Ou ainda, num intervalo de tempo específico, com determinada característica atribuída (Becker \& Razzouk, 2018; Daltio, Mari \& Ferraz, 2011). Os dados foram observados de forma retrospectiva (Razzouk et al, 2015) ou prospectiva (Chang et al, 2012), em alguns casos utilizando dados dos prontuários (Lindner, 2009; Son et al, 2019).

A esquizofrenia foi o transtorno mental mais investigado em relação à testagem de intervenções com relação ao custo da doença propriamente dito. Quando comparada a outras doenças psiquiátrica obteve maior impacto de custo nos serviços de saúde (Percudani et al, 2004). Ainda nesta direção, observa-se que, quanto maior o avanço da gravidade dos transtornos mentais, maior a despesa gerada em relação à oferta do cuidado (Medina et al, 2018). Nesta despesa está inserido o custo com intervenções medicamentosas que foram calculadas através do perfil de utilização individual de cada sujeito da pesquisa (Tait et al, 2016; Ekman et al, 2013; Razzouk et al, 2015). Outras despesas também foram consideradas; entre elas a despesa com o salário dos profissionais de saúde, que representou o maior custo financeiro quando avaliada em serviço comunitário de saúde (Becker \& Razzouk, 2018).

A mensuração e o método de análise corresponderam à perspectiva e ao tipo de custo que foram avaliados. Foram identificadas avaliações mais complexas como a modelagem econômica, indicando a estimativa de cenários mais propícios a intervenção e a relação custo-benefício, como por exemplo: a utilização de Risperidona e Haloperidol antes da Olanzapina no tratamento da esquizofrenia (Lindner et al, 2009).

\section{Conclusão}

Estudos de análise econômica em saúde têm sido realizados em cenários assistenciais públicos e da saúde mental. 
Envolvem perspectivas variadas conforme o objetivo de cada pesquisa, demonstrando valores financeiros relativos aos transtornos e ao cuidado ofertado, seja de forma direta ou indireta.

Nesta revisão integrativa, o custo direto médico hospitalar foi mais elevado nos estudos analisados. Além disso, identificou-se com mais elevado custo as variáveis: medicalização, diárias de internação e folha salarial. A maneira de contabilização dessas despesas foi identificada através de informações relacionadas ao perfil de utilização do serviço, recursos estruturais e recursos humanos institucionais, considerando a quantidade de usuários ou sujeitos participantes da pesquisa.

Identificou-se ainda que, os serviços hospitalares que oferecem internação psiquiátrica produzem maior custo direto médico hospitalar. O uso da Risperidona injetável mostrou-se relevante em relação ao custo-benefício, tendo em vista que, quando o paciente se encontra estável sem necessidade de internação, ele possivelmente utilizará mais o serviço comunitário do que o hospital. A esquizofrenia foi o transtorno mental mais investigado. Quanto maior a gravidade dos transtornos mentais, maior a despesa gerada em relação à oferta do cuidado.

Embora seja crescente o desenvolvimento de tais estudos em ambientes comunitários, assistenciais e públicos de saúde, percebe-se que tem sido predominante a investigação econômica na conjuntura do cuidado em saúde mental na fase adulta. Existe então, uma lacuna a ser preenchida por estudos que abordem o custo público com cuidados em saúde mental voltado a crianças e adolescentes.

Desta maneira sugere-se que novos estudos quantitativos de análise de custo possam ser realizados direcionados a esse público e que contemplem avaliação das despesas desde o funcionamento dos serviços até a oferta da assistência em saúde mental propriamente dita.

Esta revisão contemplou apenas estudos provenientes de análise de custo realizadas em serviços públicos de saúde mental. Estes estudos nem sempre são especificamente direcionados a intervenções, o que resultou em poucos estudos de ensaios clínicos randomizados.

\section{Referências}

Amaddeo, F., Beecham, J., Bonizzato, P., Fenyo, A., Knapp, M. \& Tansellaet, M. (1997). The use of a case register to evaluate the costs of psychiatric: care. Actu Psychiatr Scund. 95, 189-98

Becker, P \& Razzouk, D. (2018). Cost of a community mental health service: a retrospective study on a psychosocial care center for alcohol and drug users in São Paulo. São Paulo Med J. 136(5):433-41.

Chang, H. C., Tang, C. H., Huang, S. T., McCrone, P. \& Su, K. P. (2012). A cost-consequence analysis of long-acting injectable risperidone in schizophrenia: A one-year mirror-image study with national claim-based database in Taiwan. Journal of Psychiatric Research. 46, 751-6.

Daltio, C. S., Mari, J. J. \& Ferraz, M. B. (2011). Direct medical costs associated with schizophrenia relapses in health care services in the city of São Paulo. Rev Saude Publica, 45(1):14-23.

Ekman, M., Granström, O., Omérov, S., Jacob, J. \& Landén M. (2013). The societal cost of bipolar disorder in Sweden. Soc Psychiatry Psychiatr Epidemiol.

Fineout-Overholt, E. \& Stillwell, S. B. (2011). Asking compelling, clinical questions. In: Melnyk BM, Fineout-Overholt E. Evidence-based practice in nursing \& healthcare: a guide to best practice. Philadelphia: Wolters Kluwer, Lippincot Williams \& Wilkins. 25-39.

Galvão, C. M. (2006). Níveis de Evidência. Acta. Paul. Enferm. 19 (2).

Garattini, L., Rossi, C., Tediosi, F., Cornaggia, C., Covelli, G., Barbui, C. \& Parazzini, F. (2001). Direct Costs of Schizophrenia in Italian Community Psychiatric Services. Pharmacoeconomics. 19 (12): 1217-1225.

Itria, A., Novaes, H. M. D., Soárez, P.C., Nobrega, L. A. L., Sartori, A. M. C. (2012). A importância dos métodos de custeio e valoração nas avaliações econômicas em saúde: repercussões sobre os resultados de avaliação da vacina antimeningocócica C. Physis [Internet]. 22(2): 641-658.

Lindner, L. M., Marasciulo, A. C., Farias, M. R \& Grohs, G. E. M. (2009). Avaliação econômica do tratamento da esquizofrenia com antipsicóticos no Sistema Único de Saúde. Rev Saúde Pública, 43(1):62-9.

Mackotha, Z., Saravana, K. \& Perraton, L. G. (2009). A systematic review of the literature on the effectiveness of exercise therapy for groin pain in athletes. Sports Medicine, Arthroscopy, Rehabilitation, Therapy \& Technology. .1 (5)

Medina, M., Velásquez,, A.V., Ribero, O. J. \& Trujillo, N. (2018) Costos médicos directos por intento de suicidio en pacientes del hospital mental de Antioquia, Colombia. Rev Panam Salud Publica. 42, 1-8. 
Moher, D., Liberati, A., Tetzlaff, J. \& Altman, D. G. (2009). Preferred Reporting Items for Systematic Reviews and Meta-Analyses: The PRISMA Statement. PLoS Med, 6(7): e1000097.

Organização Mundial da Saúde. (2020). Dia Mundial da Saúde Mental: uma oportunidade para dar o pontapé inicial em uma grande escala de investimentos.

Onocko-Campos, R. T. (2019). Saúde mental no Brasil: avanços, retrocessos e desafios. Cad. Saúde Pública. 35(11):e00156119.

Percudani, M., Barbui C., Beecham, J. \& Knapp, M. (2004). Routine outcome monitoring in clinical practice: service and non-service costs of psychiatric patients attending a Community Mental Health Centre in Italy. European Psychiatry. 19, 469-477.

Razzouk, D., Kayo, M., Sousa, A., Gregorio, G., Cogo-Moreira, H., Cardoso, A. A. \& Mari, J. J. (2015). The Impact of Antipsychotic Polytherapy Costs in the Public Health Care in Sao Paulo, Brazil. Plos One. 10 (4):1-13.

Ruggeri, M., Bisoffi, G., Lasalvia, A., Amaddeo, F., Bonetto, C. \& Biggeriet, A. (2004). A longitudinal evaluation of two-year outcome in a community-based mental health service using graphical chain models. International Journal of Methods in Psychiatric Research. 13(1):10-23.

Salize, H. J., McCabe, R., Bullenkamp, J., Hansson, L., Lauber, C., Martinez-Leal, R. \& Reinhard, I. (2009).Cost of treatment of schizophrenia in six European countries. Schizophrenia Research. 111, 70-77.

Santos, C. M. C., Pimenta, C. A. M. \& Nobre, M. R. C. (2007). A estratégia PICO para a construção da pergunta de pesquisa e busca de evidências. Rev Latinoam Enfermagem. 15(3).

Silva, E. N., Silva, M. T. \& Pereira, M. G. (2016). Identificação, mensuração e valoração de custos em saúde. Epidemiol. Serv. Saude. 25(2):437-439.

Son, J. M., Juncal-Ruiz, M., Foz, V. O. G., Cantarero-Prieto, D., Blázquez-Fernández, C., Paz-Zulueta, M. \& et al. (2019). Understanding the direct and indirect costs of a first episode of psychosis program: Insights from PAFIP of Cantabria, Spain, during the first year of intervention. Early Intervention in Psychiatry. $13,1182-1190$.

Tait, R. J., Teoh, L., Kelty, E., Geelhoed, E., Mountain, D. \& Hulse, G. K. (2016). Emergency department based intervention with adolescent substance users: 10 year economic and health outcomes. Drug and Alcohol Dependence. 165, 168-174.

Tarricone, R., Gerzeli, S., Montanelli, R., Frattura, L., Percudani, M. \& Racagni, G. (2000). Direct and indirect costs of schizophrenia in community psychiatric services in Italy The GISIES study. Health Policy. 51, 1-18.

Whittemore, R. \& Knafl, K. (2005). The integrative review: updated methodology. Blackwell Publishing Ltd,Journal of Advanced Nursing. 52(5): 546-553.

Wiersma, D., Kluiter, H., Nienhuis, F. J., Rüphan, M., Giel, R. \& et al. (1991).Costs and Benefits of Day Treatment With Community Care for Schizophrenic Patients. Schizophrenia Bulletin. 17(3): 411-419. 